\section{Zur Biogenese des Cyanidins}

II. Mitt.: Über die Brompikrinspaltung des Trinitrophloroglucins

Von Hans Grisebach

Organisch-chemisches Institut der Technischen Universität Berlin-Charlottenburg

(Z. Naturforschg. 12 b, 597-598 [1957]; eingegangen am 18. Juni 1957)

In der ersten Mitt. ${ }^{1}$ wurde u. a. über den Abbau des radioaktiven Cyanidins berichtet. Das durch Alkalischmelze aus dem Cyanidin erhaltene Phloroglucin wurde dabei in Trinitrophloroglucin übergeführt und dieses mit Bariumhypobromid in Tribromnitromethan und Kohlendioxyd gespalten. Nach dem Ergebnis der Brompikrinspaltung bei der Pikrinsäure ${ }^{2}$ wäre hierbei zu erwarten gewesen, daß das gebildete Kohlendioxyd ausschließlich aus den C-Atomen 2, 4 und 6 des Trinitrophloroglucins * stammt, während das Tribromnitromethan aus den C-Atomen 1, 3 und 5 entsteht. Dies wurde nun mit Hilfe von synthetischem Trinitrophloroglucin- $\left[2.4 .6-{ }^{14} \mathrm{C}\right]$ geprüft. Phloroglucin-[1.3.5$\left.{ }^{14} \mathrm{C}\right]$ erhielt man aus Malonester- $\left[1-{ }^{14} \mathrm{C}\right]$ nach einer Synthese von BAEYER ${ }^{3}$ durch Kondensation mit Na und anschließende Decarboxylierung des dabei gebildeten Phloroglucin-dicarbonsäureesters. Die Brompikrinspaltung wurde mit Calciumhypobromid ${ }^{4}$ oder Bariumhypobromid ${ }^{2}$, in einer Acetyl-Bestimmungs-Apparatur nach $\mathrm{W}_{\text {IESENBERger }}{ }^{5}$ ausgeführt. Diese Apparatur eignet sich besonders gut für den Abbau, da das gebildete Tribromnitromethan gleich durch eine 3 -fache Wasserdampfdestillation gereinigt wird. Nach weiterer Reinigung durch mehrfaches Waschen mit Wasser wurde das Tribromnitromethan im Platinschiffchen im Luftstrom verbrannt und das $\mathrm{CO}_{2}$ im Gaszählrohr gemessen. Das während der Brompikrin-Reaktion gebildete $\mathrm{CO}_{2}$ erhält man durch Ansäuern der Reaktionslösung. Um dabei freies Brom zurückzuhalten, wird beim Überspülen des
$\mathrm{CO}_{2}$ mit Stickstoff in eine Waschflasche mit Natronlauge eine Waschflasche mit Phenol von $50^{\circ}$ dazwischen geschaltet. Tab. 1 zeigt die Ergebnisse der radioaktiven Messungen.

Die Ergebnisse zeigen, daß die spez. Aktivität des Kohlendioxyds um 25 bis $30 \%$ zu niedrig liegt, wenn man den Abbau unter den Bedingungen von EHrENSVÄRD ${ }^{2}$ in der Siedhitze durchführt. Verzichtete man auf das Abdestillieren des Tribromnitromethans und ließ die Reaktion bei $70^{\circ}$ ablaufen, so lag die spez. Aktivität des $\mathrm{CO}_{2}$ nur noch um $17 \%$ zu niedrig. Man könnte zunächst annehmen, daß die Synthese des Phloroglucins- $\left[1.3 .5-{ }^{14} \mathrm{C}\right]$ nicht eindeutig verlaufen ist und sich auch Aktivität in den C-Atomen 1, 3 und 5 befindet. Dies ist aber nicht der Fall, da das gebildete Tribromnitromethan (Ausbeute etwa 60\%) inaktiv ist. Man kann die zu geringe spez. Aktivität des $\mathrm{CO}_{2}$ daher nur so erklären, daß bei der Reaktion auch ein Teil der C-A tome 1, 3 und $5 \mathrm{zu} \mathrm{CO}_{2}$ oxydiert werden und somit eine Verdünnung des radioaktiven $\mathrm{CO}_{2}$ eintritt. Diese Oxydation ließe sich möglicherweise vermeiden, wenn man die Reaktion bei tieferer Temperatur durchführt. Wegen Mangel an radioaktivem Trinitrophloroglucin konnte dies nicht mehr geprüft werden. Auf jeden Fall erhält man richtige Werte für die Aktivitätsverteilung im Phloroglucinring, wenn man sich nur auf die Aktivität des Tribromnitromethans bezieht, da dieses eindeutig aus den C-Atomen 1, 3 und 5 stammt.

Nach Birch und Moye ${ }^{6}$ entsteht bei der BrompikrinReaktion mit $o-, m$ - und $p$-Nitrophenol neben dem Tribromnitromethan auch Bromoform, was ir-spektroskopisch nachgewiesen wurde. Das Tribromnitromethan aus dem Trinitrophloroglucin enthält nach dem IRSpektrum kein Bromoform.

Da die in der ersten Mitt. ${ }^{1}$ veröffentlichten Werte für die Aktivität der C-Atome 2, 4 und 6 des Trinitrophloroglucins aus dem Cyanidin aus einem BrompikrinAbbau mit Bariumhypobromid bei Siedehitze erhalten

\begin{tabular}{|c|c|c|c|c|}
\hline $\begin{array}{c}\text { Trinitrophlorglucin } \\
\text { [Imp /Min./mMol] }\end{array}$ & $\begin{array}{c}\text { Kohlendioxyd } \\
\text { [Imp./Min./mMol] }\end{array}$ & $\begin{array}{c}\text { Aktivität von } \\
\text { Trinitrophlorglucin [\%] }\end{array}$ & $\begin{array}{c}\text { Tribromnitromethan } \\
\text { [Imp./Min./mMol] }\end{array}$ & Temp. \\
\hline 31039 & 23800 & 75 & keine & Siedehitze \\
30810 & 21800 & 71 & - &, \\
15873 & 11050 & 70 & - & $70^{\circ}$ \\
15873 & 13650 & 86 & $4 \pm 2$ & \\
\hline
\end{tabular}

Tab. 1. Brompikrin-Reaktion mit Trinitrophlorglucin- $\left[2.4 \cdot 6^{-14} \mathrm{C}\right]$.

1 H. Grisebach, Z. Naturforschg. 12 b, 227 [1957].

2 L. Reio u. G. Ehrensvärd, Ark. Kemi 5, 301 [1953].<smiles>O=[N+]([O-])c1c(O)c([N+](=O)[O-])c(O)c([N+](=O)[O-])c1O</smiles>

3 A. Baeyer, Ber. dtsch. chem. Ges. 18, 3454 [1885]. wurden, bedürfen diese Zahlen einer Korrektur. Die Abbauten wurden wiederholt und die RadioaktivitätsVerteilung im Phloroglucinring nur aus der Aktivität des Tribromnitromethans berechnet. Die neuen Meßergebnisse sind in Tab. 2 zusammengestellt.

${ }^{4}$ S. Aranoff, Techniques of Radiobiochemistry, S. 189, Iowa State College Press 1956.

5 E. Wiesenberger, Mikrochem. verein. Mikrochim. Acta 33, 51, [1948].

6 A. J. Birch u. C. I. Moye, Privatmitteilung. 


\begin{tabular}{|c|c|c|c|c|}
\hline \multicolumn{3}{|c|}{ Versuch mit Acetat- $\left[1-^{-14} \mathrm{C}\right]$} & \multicolumn{2}{|c|}{ Versuch mit Acetat- $\left[2-^{-14} \mathrm{C}\right]$} \\
\hline [Imp./Min & & $\begin{array}{c}\text { Aktivität von } \\
\text { Trinitrophlorglucin } \\
{[\%]}\end{array}$ & [Imp./Min./mMol] & $\begin{array}{c}\text { Aktivität von } \\
\text { Trinitrophlorglucin } \\
{[\%]}\end{array}$ \\
\hline $\begin{array}{l}\text { Trinitrophlorglucin } \\
\mathrm{Br}_{3} \mathrm{CNO}_{2} \\
\left(\mathrm{C}_{1,3,5}\right) \\
\mathrm{C}_{2,4,6}\end{array}$ & $\begin{aligned} 836 & \pm 18 \\
16 & \pm 5 \\
& -\end{aligned}$ & $\begin{array}{r}100 \\
2 \\
98\end{array}$ & $\begin{array}{l}1285 \pm 26 \\
1071 \pm 25\end{array}$ & $\begin{array}{r}100 \\
83 \\
17\end{array}$ \\
\hline
\end{tabular}

Tab. 2. Brompikrinabbau mit Trinitrophloroglucin aus Cyanidin.

Die Aktivität der C-Atome 2, 4 und 6 erhielt man nur durch Differenzbildung, was aber bei der Genauigkeit der Aktivitätsmessung in der Gasphase zulässig ist. Die neu erhaltenen Werte zeigen noch eindeutiger als die verfälschten Werte der ersten Mitt., daß die eine Sauerstoff-Funktion tragenden C-Atome des Phloro- glucinringes des Cyanidins aus den Carboxylgruppen und die anderen 3 C-Atome aus den Methylgruppen des Acetats stammen.

Herrn Professor Weygand danke ich für die Förderung dieser Arbeit. Weiterhin danke ich Herrn G. Biessalski für die radioaktiven Messungen.

\section{Über den Stoff wechsel von meso-Inosit}

Von H. Herken, D. Maibauer und F. Weygand

Pharmakol. Institut der Freien Universität Berlin und Organ.-chem. Institut der Techn. Universität Berlin (Z. Naturforschg. 12 b, 598-599 [1957]; eingegangen am 11. Juli 1957)

In den bisherigen Untersuchungen über den Stoffwechsel von meso-Inosit wurde die kontinuierliche Freisetzung dieses Stoffes aus Schnitten von Gehirngewebe durch Herken und Maibauer ${ }^{1}$ nachgewiesen. Weiterhin konnte die intrazelluläre Verteilung des meso-Inosits bestimmt werden, wobei eine Anreicherung im Zellplasma, besonders des Gehirns, gegenüber dem Blut gefunden wurde ${ }^{2}$. Diese Untersuchungen wurden jetzt mit Hilfe von uniform ${ }^{14} \mathrm{C}$-markiertem meso-Inosit, der von Weygand und $\mathrm{S}_{\text {chulze }}{ }^{3}$ synthetisiert wurde, nachgeprüft und erweitert.

Einer männlichen Ratte von $250 \mathrm{~g}$ Gewicht wurde $1 \mathrm{mg}{ }^{14} \mathrm{C}$-markierten meso-Inosits in wäßriger Lösung i.v. injiziert und 24 Stdn. später die Zellen von Gehirn und Leber in der früher beschriebenen Weise fraktioniert und neben der Menge meso-Inosit/mg N auch die Aktivität/mg N in den Fraktionen bestimmt.

Bei der Aufarbeitung der Zellfraktionen stellte sich heraus, daß die Aktivität $0,1-1,6 \%$ der gesamten Inositmenge in der jeweiligen Zellfraktion entsprach (Tab. 1). Das Verhältnis der Aktivität der einzelnen Zellfraktio-

\begin{tabular}{|c|c|c|c|c|c|c|}
\hline \multirow{2}{*}{ Organ } & \multirow{2}{*}{ Zellfraktion } & \multirow{2}{*}{$\gamma$ meso-Inosit/mg $\mathrm{N}$} & \multirow{2}{*}{$\begin{array}{l}\text { Aktivität entsprech. } \\
\gamma \text { meso-Inosit-14 } \mathrm{C} / \\
\mathrm{mg} \mathrm{N}\end{array}$} & \multirow{2}{*}{$\begin{array}{l}\text { Aktivität entsprech. } \\
\% \text { meso-Inosit-14 } \mathrm{C} \text {. } \\
\text { Gesamt-Inositmenge }\end{array}$} & \multicolumn{2}{|c|}{ Mengen bezogen auf Kernfraktion $=1$, } \\
\hline & & & & & meso-Inosit & $\begin{array}{l}\text { Aktivität entsprech. } \\
\text { meso-Inosit-14 } \mathrm{C}\end{array}$ \\
\hline Leber & $\begin{array}{l}\text { Kerne } \\
\text { Mitochondrien } \\
\text { Mikrosomen } \\
\text { Plasma }\end{array}$ & $\begin{array}{r}4 \\
15 \\
32 \\
9\end{array}$ & $\begin{array}{l}0,04 \\
0,19 \\
0,36 \\
0,14\end{array}$ & $\begin{array}{l}1,0 \\
1,3 \\
1,1 \\
1,6\end{array}$ & $\begin{array}{l}1 \\
3,75 \\
8,0 \\
2,25\end{array}$ & $\begin{array}{l}1 \\
4,75 \\
9,0 \\
3,5\end{array}$ \\
\hline Gehirn & $\begin{array}{l}\text { Kerne } \\
\text { Mitochondrien } \\
\text { Mikrosomen } \\
\text { Plasma }\end{array}$ & $\begin{array}{r}37 \\
18 \\
35 \\
137\end{array}$ & $\begin{array}{l}0,04 \\
0,04 \\
0,1 \\
0,33\end{array}$ & $\begin{array}{l}0,1 \\
0,2 \\
0,3 \\
0,2\end{array}$ & $\begin{array}{l}1 \\
0,5 \\
1,0 \\
3,7\end{array}$ & $\begin{array}{l}1 \\
1 \\
2,5 \\
8,25\end{array}$ \\
\hline
\end{tabular}

Tab.l. Die Bestimmung des meso-Inosits erfolgte mikrobiologisch in der bereits beschriebenen Weise (MaIbaUer und Herken ${ }^{2}$ ) mit Hilfe von Saccharomyces Carlsbergensis 4228 ATCC 9080, die ${ }^{14} \mathrm{C}$-Bestimmung durch Trockenverbrennung und Messung der $\mathrm{CO}_{2}$-Aktivität im Gaszählrohr (F. WeYGAND, Naturwissenschaften 44, 169 [1957]).

1 H. Herken u. D. Matbauer, Naturwissenchaften 42, 166 [1954].
2 D. Maibauer u. H. Herken, Naunyn-Schmiedebergs Arch. exp. Pathol. Pharmakol. 227, 456 [1956].

3 F. WeYGand u. E. Schulze, Z. Naturforschg. 11 b, 370 [1956]. 